\title{
MEUDON GRAVITATIONAL RADIATION DETECTION EXPERIMENT
}

\author{
S. BONAZZOLA, M. CHEVRETON and J. THIERRY-MIEG \\ Observatoire de Paris, Meudon, France.
}

\begin{abstract}
A description of Weber's type experiment of gravitational waves detection is given. Double and triple coincidences with Frascati and Munich groups were looked for with negative results. A capacitive passive method of detection has been presented. This method can improve the sensitivity by a factor of 30 when working at room temperature.
\end{abstract}

